\title{
CORRELATION BETWEEN MECHANISM OF INJURY AND TRAUMATIC LESIONS ON PELVIC TRAUMA.
}

\section{Ricardo R. G. de Almeida*, Thiago R. A. Calderan, Gustavo P. Fraga}

\section{Abstract}

Pelvic trauma is responsible for high complexity and morbidity lesions; especially bleeding and infection. 38\% of all trauma involves scenarios tippically associated with pelvic trauma. This study aimed to correlate the admission data of the patients, the mechanism of injury, the traumatic lesions and outcomes.

\section{Key words:}

Pelvic trauma, mechanism of injury, traumatic lesions

\section{Introduction}

Trauma is the major death cause in adults between 15-29 years. Nearly $38 \%$ involves vehicle collision, trampling and fall from height; all of them related to pelvic trauma.

Pelvic trauma is associated with high complexity and morbidity injuries, being bleeding the major cause of mortality in the first 24 hours. Also, the pelvic cavity contents wounds are likely to progress to infection and sepsis or chronic complications such as urinary or faecal incontinence and sexual dysfunctions.

Identifying this condition is hard without complementary studies, bringing importance to the information about the mechanism of injury on the admission of these patients in low complexity centers.

This study aimed to correlate mechanism of injury, traumatic lesions, trauma scores and patient outcome.

\section{Results and Discussion}

Records of 65 patients were included on this study. The inclusion criteria were: been admitted with pelvic trauma on HC UNICAMP Intensive Care Unit between 01/01/2010 and $12 / 31 / 2016$, have more than 14 years old and with admission data clearly registered on medical records.

The patients were spread across three groups according to the mechanism of injury: MOT (motorcycle collision), CAR (car collision), TRA (hit by a vehicle), FAL (fall from height), BUS (collision or run over by a bus or truck) and SMA (crushing by heavy objects).

Chart 1. Means and Standard deviation of variables in each group.

\begin{tabular}{ccccccc}
\hline & $\begin{array}{c}\text { MOT } \\
(n=21)\end{array}$ & $\begin{array}{c}\text { CAR } \\
(n=13)\end{array}$ & $\begin{array}{c}\text { TRA } \\
(n=18)\end{array}$ & $\begin{array}{c}\text { FAL } \\
(n=7)\end{array}$ & $\begin{array}{c}\text { BUS } \\
(n=3)\end{array}$ & $\begin{array}{c}\text { SMA } \\
(n=3)\end{array}$ \\
\hline Age (years) & $31,29 \pm 11,14$ & $36,00 \pm 13,67$ & $41,17 \pm 15,99$ & $31,43 \pm 12,22$ & $29,00 \pm 11,00$ & $59,00 \pm 13,77$ \\
Hospital Stay (days) & $35,24 \pm 41,20$ & $24,54 \pm 24,9$ & $27,22 \pm 22,74$ & $59,00 \pm 73,79$ & $25,33 \pm 17,16$ & $22,00 \pm 26,46$ \\
Number of injuries & $6,52 \pm 2,29$ & $6,69 \pm 2,10$ & $5,61 \pm 2,87$ & $6,14 \pm 2,61$ & $5,67 \pm 0,58$ & $5,33 \pm 3,21$ \\
Red Blood Cells (packs) & $3,45 \pm 5,11$ & $1,31 \pm 2,43$ & $2,39 \pm 1,94$ & $0,57 \pm 0,98$ & $5,00 \pm 8,66$ & $4,33 \pm 4,93$ \\
RTS & $5,74 \pm 2,62$ & $5,76 \pm 2,41$ & $5,37 \pm 2,49$ & $5,7 \pm 2,18$ & $6,15 \pm 2,32$ & $3,98 \pm 2,74$ \\
ISS & $32,38 \pm 14,49$ & $29,08 \pm 9,48$ & $29,67 \pm 11,47$ & $25,86 \pm 8,34$ & $27,00 \pm 6,24$ & $24,00 \pm 10,54$ \\
NISS & $37,00 \pm 13,39$ & $31,23 \pm 11,20$ & $34,28 \pm 9,52$ & $27,86 \pm 7,24$ & $30,00 \pm 6,93$ & $30,00 \pm 6,93$ \\
TRISS & $0,64 \pm 0,38$ & $0,68 \pm 0,34$ & $0,63 \pm 0,35$ & $0,80 \pm 0,18$ & $0,85 \pm 0,19$ & $0,40 \pm 0,45$ \\
NTRISS & $0,61 \pm 0,40$ & $0,65 \pm 0,34$ & $0,60 \pm 0,37$ & $0,78 \pm 0,20$ & $0,83 \pm 0,18$ & $0,36 \pm 0,43$ \\
Infection & $47,60 \%$ & $38,50 \%$ & $55,60 \%$ & $28,60 \%$ & $33,30 \%$ & $0,00 \%$ \\
Death & $28,60 \%$ & $23,10 \%$ & $27,80 \%$ & $0 \%$ & $33,30 \%$ & $33,30 \%$ \\
\hline
\end{tabular}

The Number of Injuries stand for the number of coded AIS (Abbreviated Injury score) injuries the patient got on every segment added up. It doesn't count the score of each injury.
There was no statistically significant difference of age, hospital stay, RTS (Revised Trauma Score), ISS, NISS, TRISS, NTRISS, infection and death between each group. However, there was on number of injuries (nINJ) and number of red blood cells (nRBC) packs.

The nRBC was significantly higher $(p<0,05)$ on TRA group over FAL; having no difference between other groups. Additionally, the nINJ was significantly higher $(\mathrm{p}<$ 0,05 ) in MOT and TRA over FAL, BUS and SMA. Also, the nINJ on CAR was higher than on BUS and SMA.

Despite the difference among the nRBC in each group, there were no divergence in "death"; even with a positive relation between $n R B C$ and death $(p<0,05)$.

The nINJ contrast, brings a discussion as well; since it disagrees with the non-existence divergence of ISS or NISS between groups. This could mean that although the number is higher, the severity of the injuries was higher in BUS and SMA.

Digital rectal examination found hypotonic sphincter with $100 \%$ Sensibility and $100 \%$ Specificity on the 4 patients that developed urinary incontinence on the follow-up.

Unstable pelvis finding on physical examination did not showed a correlation with death, nRBC, nINJ or hospital stay; however, we identified an association $(p<0,05)$ with Infection.

\section{Conclusions}

This study was not enough to provide robust conclusions over pelvic trauma and mechanism of injury. The sample size was the greatest difficulty to achieve statistically significant information.

A new project with a bigger scale and, preferably, prospective methodology is necessary.

1. American College of Surgeons (2018). Advanced Trauma and Life Support. 10th ed.

2. World Health Organization. Injuries and violence: the facts 2014. 2014.

3. Coccolini F, Stahel PF, Montori G, Biffl W, Horer TM, Catena F, et al. Pelvic trauma: WSES classification and guidelines. World Journal of Emergency Surgery (2017) 12:5.

4. Choi W, Rhee H, Chung E. Lower urinary tract imaging in pelvic fracture: an 11-year review of genitourinary complications and clinical outcomes. ANZ J Surg. 2016 Dec 1.

5. Wang H, Robinson RD, Moore B, Kirk AJ, Philips JL, Umejiego J, et al. Predictors of early versus late mortality in pelvic trauma patients. Scand J Trauma Resusc Emerg Med. 2016 Mar 10;24:27. 